\title{
Lack of reacquisition in learned taste aversions
}

\author{
JABER DANGUIR and STYLIANOS NICOLAIDIS \\ Laboratoire de Neurobiologie des Régulations, Collège de France, 75231 Paris, France
}

\begin{abstract}
Following ingestion of either water (Experiment 1) or saccharin (Experiment 2), experimental groups of rats were poisoned with lithium chloride and acquired an aversion to the ingested fluid. This aversion gradually extinguished and, in both experiments, was not reacquired when fluid intake was again followed by poisoning. These results are in marked contrast to usual findings of very rapid relearning following extinction with conditioning preparations other than taste-aversion learning.
\end{abstract}

When a distinctive flavor is followed by toxicosis, animals generally subsequently evidence an aversion for that flavor. This taste-aversion learning exhibits several features which distinguish it from more traditional learning preparations (see Revusky \& Garcia, 1970), and some investigators have suggested that such flavor-illness associations are a distinct, biologically specialized form of learning (e.g., Rozin \& Kalat, 1971). However, other investigators have suggested that the general principles underlying taste-aversion learning and other forms of learning may be similar, with the dramatic differences being primarily of a quantitative nature (e.g., Best, 1975).

The results of the present experiments suggest one qualitative difference between taste-aversion learning and other forms of learning. It has been demonstrated in a variety of conditioning situations that the reinstatement of a conditional response following extinction is relatively rapid. That is, a response which has been classically conditioned through repeated conditioned stimulus (CS)-unconditioned stimulus (UCS) pairings, then extinguished by CS-alone presentations, is usually reestablished by CS-UCS pairings much more quickly than if there were no prior history of CS-UCS pairings (e.g., Ellson, 1938; Pavlov, 1927, p. 59). In contrast with these findings, the results of the present experiments, using the tasteaversion situation, indicated that prior acquisitionextinction experience did not facilitate reacquisition -indeed, it profoundly retarded reacquisition.

We have previously reported experiments concerning the acquisition and extinction of an aversion to water, based on lithium chloride $(\mathrm{LiCl})$ toxicosis (Danguir, 1975). In Experiment 1 of the present paper, the postextinction reacquisition of such a learned water aversion was investigated. In Experi-

The authors are grateful to N. E. Rowland and P. Rozin for criticisms of an earlier draft of this manuscript, and to $\mathrm{Dr}$. $\mathrm{S}$. Siegel for his editorial assistance. Requests for reprints should be addressed to Jaber Danguir, Laboratoire de Neurobiologie des Régulations, College de France, 11 place Marcellin Berthelot, 75231 Paris Cedex 05, France. ment 2 , the reacquisition of a learned saccharinaversion was assessed.

\section{EXPERIMENT 1}

\section{Method}

Twenty male rats of the Wistar strain were individually housed with food ("Extralabo M 25") available ad lib. Tap water was available only from 10:00 to 10:15 a.m. daily. The experiment was conducted over a 23-day period. The rats were adapted to the drinking schedule for the first 8 days. Days 9-11 constituted the acquisition phase of the experiment. Five minutes after water ingestion, half the rats (experimental group, E) were injected with $\mathrm{LiCl}$ ( $2 \%$ body weight of a .15 M solution), while the remaining rats (control group, $C$ ) were injected with saline ( $2 \%$ body weight of a $.15 \mathrm{M}$ solution). The next 9 days (Days 12-20) constituted the extinction phase of the experiment: no $\mathrm{LiCl}$ was injected after the daily water-access period. On Days 21-22, both $E$ and $C$ rats received $\mathrm{LiCl}$ injections following the daily drinking sessions. Thus, Days 21 and 22 constituted reacquisition of the water aversion for $\mathrm{E}$ rats and initial acquisition for $\mathrm{C}$ rats.

To equate the groups with respect to hydrational state, each $C$ rat was yoked with an $E$ partner, such that it received a daily ration of water equal to that consumed by the $E$ partner for the first 21 days of the experiment. This ration was relatively low during acquisition, when $E$ subjects displayed a water aversion, but progressively returned to prepoisoning levels as the aversion extinguished. On Days 22 and 23, the strength of the water aversion in both $C$ and $E$ animals was assessed by measuring the amount of water freely ingested during the drinking period.

\section{Results and Discussion}

After the 8 days adaptation, $E$ rats (and thus the $C$ yoked partners) consumed a quite stable amount of water during the 15 -min presentation. Figure 1 presents the water intake results for $E$ and $C$ rats on each of Days 9-23 of the experiment. As indicated in Figure 1, $\mathrm{LiCl}$ injections on Days 9-11 caused a reduction in water consumption to approximately $2 \mathrm{ml}$ on Day 12. Thus, three water- $\mathrm{LiCl}$ pairings established a near complete suppression of intake in $\mathrm{E}$ subjects. In approximately 9 days, water consumption of $E$ rats (and thus their yoked partners) reached prepoisoning levels. When $\mathrm{LiCl}$ treatment was given to $\mathrm{E}$ and $\mathrm{C}$ groups on Day 21 , the hydrational state of all rats was similar. However, only poison-naive rats (Group C) readily developed an 


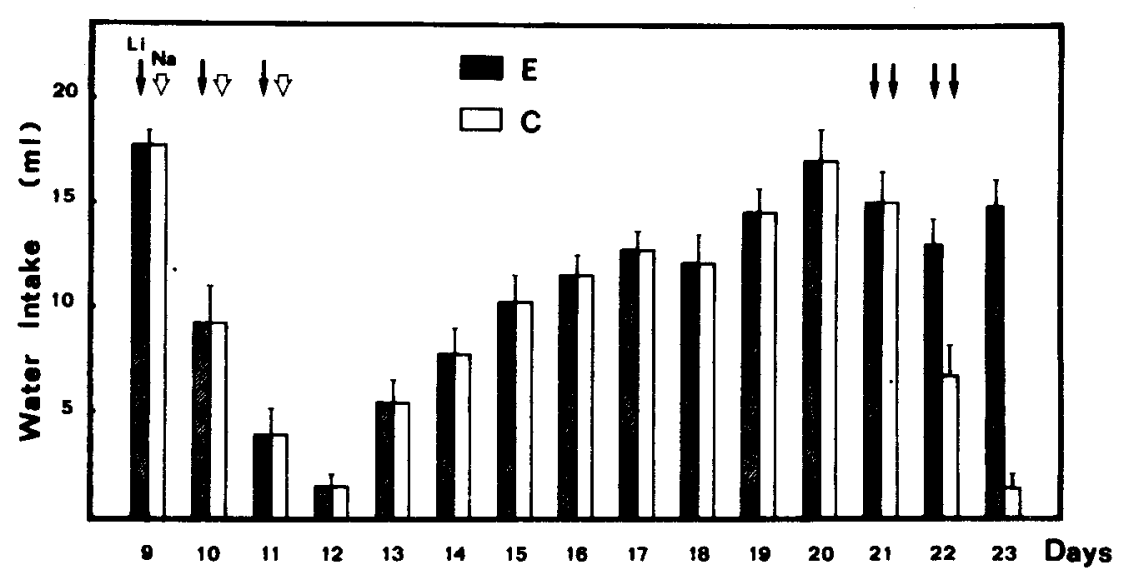

Figure 1. Water intakes (mean, standard error) of rats in Group $E$ and Group $C$ with paired fluid intakes (Experiment 1).

aversion to water, as evidenced by the substantial decrease of daily intake ( $p<.01$, Days 21 vs. 22; $t$ test); this learning was indistinguishable from that of Group E on Day 10. The aversion in Group C rats was also evidenced on Day 23 ( $p<.001$, Days 21 vs. 23). In contrast, previously poisoned Group E subjects now reduced their water intake by small and statistically insignificant amounts ( $p<.1$, Days 21 vs. 22). The difference in water consumption between the two groups was highly significant on Days $22(\mathrm{p}<.01)$ and $23(\mathrm{p}<.001)$.

The results of Experiment 1 indicated that rats which learned a tap water aversion that was subsequently extinguished (Group E) did not reacquire an aversion to water. It was noted, however, that three water- $\mathrm{LiCl}$ pairings were necessary for the establishment of a strong aversion (Days 9-11, Group E), which is in contrast to the more usual one-trial aversion, established using highly salient and/or novel tastes as the CS (Rozin \& Kalat, 1971). The failure of Group $E$ rats to acquire an aversion could be due, in part, to the familiarity and relatively low salience of tap water. This experiment could not also rule out another alternative interpretation. The absence of relearning in Group E rats may be a result of reduced novelty or effectiveness of the $\mathrm{LiCl}$ injection because of their prior experience with the toxicosis during initial acquisition (e.g., Cannon, Berman, Baker, \& Atkinson, 1975).

\section{EXPERIMENT 2}

The purpose of Experiment 2 was to determine if the failure of $\boldsymbol{E}$ rats to relearn the aversion in Experiment 1 might be attributable to either the use of water as the fluid to be avoided or the E rats' experience with $\mathrm{LiCl}$ prior to reacquisition.

\section{Method}

The CS was a $.1 \%$ saccharin solution presented for $15 \mathrm{~min}$ per day from Day 1 onwards. As in Experiment 1, water was not available in the home cages. Three different groups (n/group $=6$ ) were used. Rats in Group $\mathrm{E}_{\mathrm{S}}$ were made sick by $\mathrm{IP} \mathrm{LiCl}$ injection (same dose as Experiment 1) $5 \mathrm{~min}$ following ingestion of saccharin on Day 1. On Day 10, when the avoidance of saccharin was completely extinguished, a second such $\mathrm{LiCl}$ in jection was given.

Rats in Group $C_{S}$ were rationed to the saccharin intake of their yoked partners ( $E_{S}$ rats) until Day 10. Thus, prior to Day 11, rats of Group $C_{S}$ were in the same hydrational state as rats of Group $E_{S}$. Rats in Group $C_{S}$ received an injection of saline following saccharin ingestion on Day 1. Thus, on Day 10, the $\mathrm{LiCl}$ injection was associated with saccharin for the first time for these rats.

Rats in Group $\mathrm{C}_{\mathrm{Li}}$ were used in order to assess the effect of degradation of the US effectiveness. These rats were treated in the same manner as $\mathrm{C}_{\mathrm{S}}$ rats except that the $\mathrm{LiCl}$ injection on Day 10 was not novel, because, 2 days before the beginning of the experiment, $\mathrm{C}_{\mathrm{Li}}$ rats were injected with $\mathrm{LiCl}$ (under ad-lib food and water presentation).

\section{Results and Discussion}

Figure 2 presents the saccharin intake results for the three groups for each day of the experiment. As indicated in Figure 2, rats in Group $E_{S}$ markedly reduced their saccharin intake on Day 2 . Obviously, a single saccharin- $\mathrm{LiCl}$ pairing was sufficient to establish a near complete aversion. On subsequent days (Days 3-10), saccharin ingestion increased. On Day 10, when the aversion totally extinguished and saccharin intake had returned to or exceeded Day 1 levels, all three groups were injected with $\mathrm{LiCl}$ following saccharin access. The $E_{S}$ group did not drink significantly less saccharin on Day 11 than on Day $10(p>.1)$. In contrast, $C_{S}$ rats, made sick for the first time on Day 10, developed a strong aversion $(\mathrm{p}<.01$, Days 10 vs. 11$) . C_{\mathrm{Li}}$ rats also developed an aversion similar to that of $C_{S}$ rats; the difference of intake between the two groups on Day 11 was not significant $(p>.1)$. The aversions displayed by Groups $C_{S}$ and $C_{L i}$ were, however, less pronounced than that developed by $E_{S}$ rats on Day $2(p<.02)$. This may reflect the well-known contribution of 


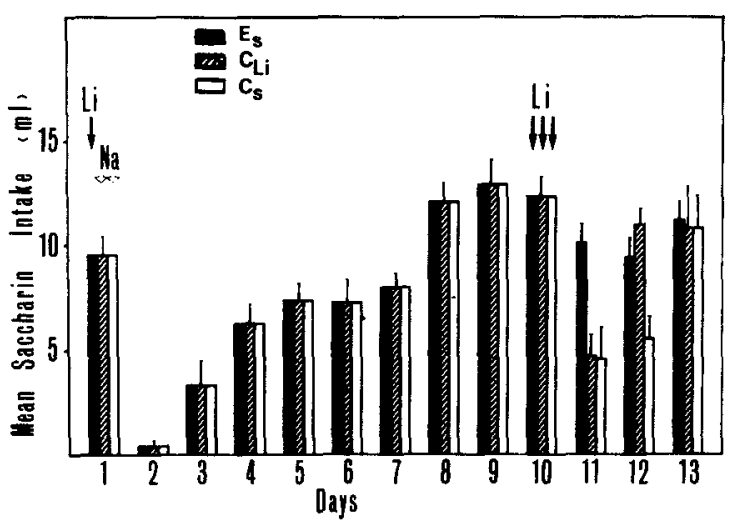

Figure 2. Saccharin intakes (mean, standard error) of rats in Groups $E_{S}, C_{L i}$, and $C_{S}$ (Experiment 2).

novelty of taste to the strength of aversion learning (e.g., Rozin \& Kalat, 1971), since, by Day 10, the saccharin was more familiar than it was on Day 1. Furthermore, the aversion formed by the $C_{\mathrm{Li}}$ and $\mathrm{C}_{\mathrm{S}}$ rats extinguished in 1 or 2 days, as indicated in Figure 2 . The $C_{L i}$ group extinguished more rapidly than the $C_{S}$ group, presumably because $C_{L i}$ rats had experienced a $\mathrm{LiCl}$ injection 2 days before the beginning of the experiment.

In this experiment, as in Experiment 1, prior acquisition and extinction precluded reacquisition (when reacquisition consisted of the same number of pairings as initial acquisition).

\section{DISCUSSION}

The results of these experiments suggest that rats have difficulty in reacquiring an extinguished taste aversion. This reacquisition deficit was demonstrated with both water (Experiment 1) and saccharin (Experiment 2) as the CS. Such failure at reacquisition is in marked contrast to results obtained from more traditional conditioning situations, and it is in agreement with suggestions that long-delay learning of taste aversions is a unique form of learning (e.g., Rozin \& Kalat, 1971).

The lack of reacquisition observed in our experiments was not due to: (a) an increased motivation of $E$ rats for drinking due to their previous several days of dehydration, since $C$ rats were equally dehydrated in the yoked design; (b) habituation of $E$ rats to the UCS, since $C_{L j}$ rats in Experiment 2 had also experienced the same dose of UCS prior to the CS-UCS pairing session on Day 10; or (c) habituation to the $\mathrm{CS}$, since all groups in our experiments were equally familiarized with the CS prior to the reacquisition phase of the experiments.

\section{REFERENCES}

Best. M. R. Conditioned and latent inhibition in taste aversion learning: Clarifying the role of learned safety. Journal of Experimental Psychology: Animal Behavior Processes, 1975, 104. 97-113.

Cannon, D. S., Berman, R. F., Baker, T. B., \& Atkinson, C. A. Effect of preconditioning unconditioned stimulus experience on learned taste aversion. Journal of Experimental Psychology: Animal Behavior Processes, 1975, 104. 270-284.

DANGUR, J. Aspects comportementaux et mécanismes neurophysiologiques de l'apprentissage à long délai chez le rat. Thèse de Doctorat de 3ème cycle, Université Paris-Sud, 1975.

Ellson, D. G. Quantitative studies of the interaction of simple habits. I. Recovery from specific and generalized effects of extinction. Journal of Experimental Psychology, 1938, 23. 339-358.

Pavlov, I. P. Conditioned reflexes. London: Oxford University Press. 1927.

Revusky, S. \& Garcia, J. Learned associations over long delays. In K. W. Spence \& J. T. Spence (Eds.), Psychology of learning and motivation. New York: Academic Press, 1970.

Rozin. P.. \& Kalat, J. W. Specific hungers and poison avoidance as adaptive specializations of learning. Psychological Review, 1971, 78, 459-485.

(Received for publication January 6, 1977; accepted June 16, 1977.) 